Supporting Information

\title{
Effect of Polymer Ligand Conformation on the Self-Assembly of Block Copolymers and Polymer-Grafted Nanoparticles within Evaporative Emulsion
}

Meng Xu ${ }^{1}$, Kang Hee Ku², Young Jun Lee ${ }^{1}$, Taewan Kim ${ }^{1}$, Jaeman J. Shin ${ }^{1}$, Eun Ji Kim ${ }^{1}$, Soo-Hyung Choi $^{3}$, Hongseok Yun ${ }^{*, 4}$ and Bumjoon J. Kim ${ }^{* 1}$

${ }^{1}$ Department of Chemical and Biomolecular Engineering, Korea Advanced Institute of Science and Technology (KAIST), Daejeon 34141, Republic of Korea

${ }^{2}$ Department of Chemical Engineering and Applied Chemistry, Chungnam National University, Daejeon 34134, Republic of Korea

${ }^{3}$ Department of Chemical Engineering, Hongik University, Seoul 04066, Republic of Korea

${ }^{4}$ Department of Chemistry, Hanyang University, Seoul 04763, Republic of Korea

*E-mail: bumjoonkim@kaist.ac.kr (B. J. K.), yunhs@hanyang.ac.kr (H. Y.) 


\section{- Supporting Figures and Tables}
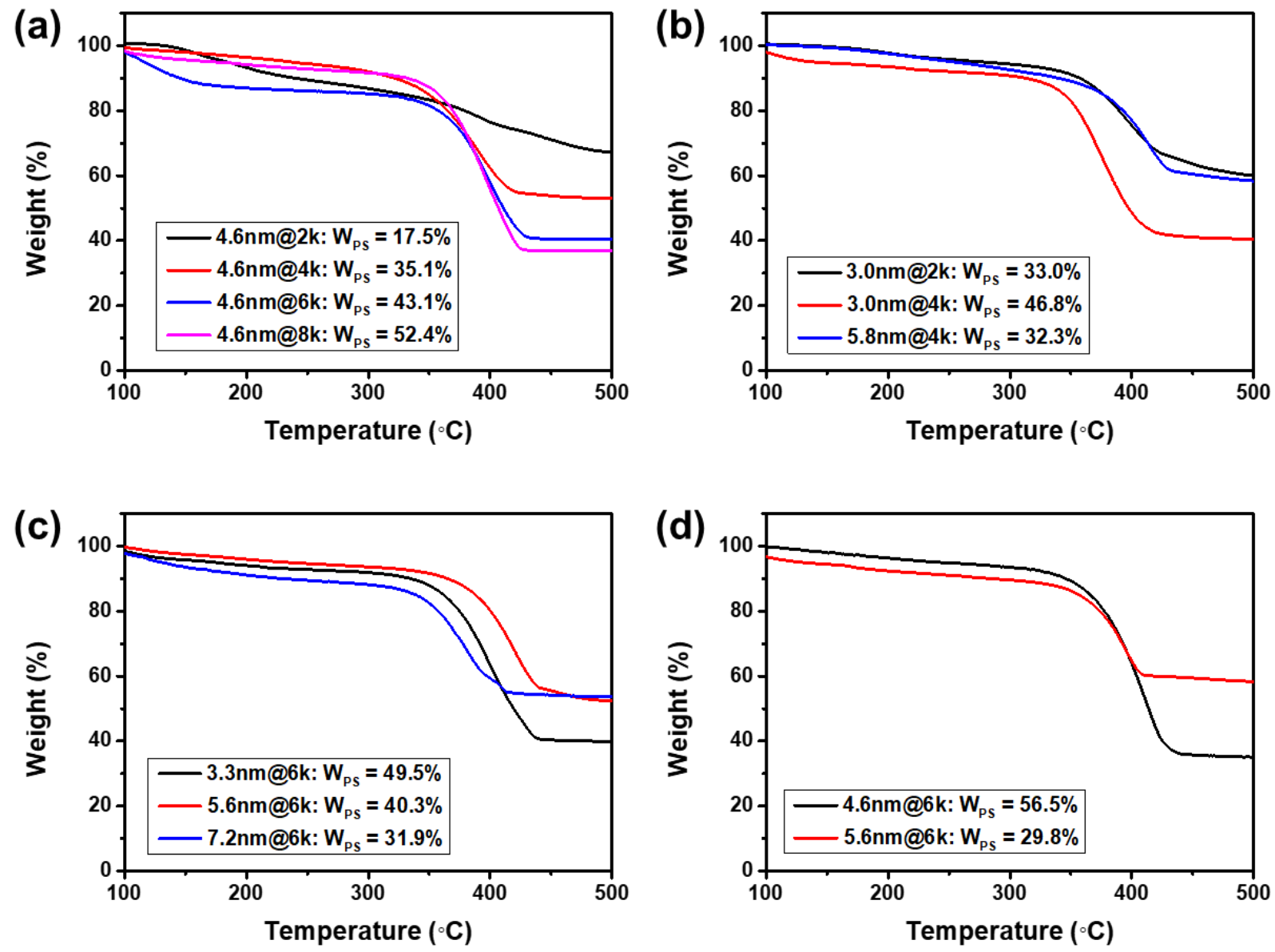

Figure S1. TGA graphs of Au@PS NPs: (a) 4.6nm@2k with $\sigma$ of 1.29 chains nm ${ }^{-2}, 4.6 \mathrm{~nm} @ 4 \mathrm{k}$ with $\sigma$ of 1.37 chains nm ${ }^{-2}, 4.6 \mathrm{~nm} @ 6 \mathrm{k}$ with $\sigma$ of 1.49 chains nm $^{-2}$, and $4.6 \mathrm{~nm} @ 8 \mathrm{k}$ with $\sigma$ of 1.58 chains nm 2; (b) 3.0nm@2k with $\sigma$ of 1.78 chains nm ${ }^{-2}, 3.0 \mathrm{~nm} @ 4 \mathrm{k}$ with $\sigma$ of 1.56 chains nm ${ }^{-2}$, and $5.8 \mathrm{~nm} @ 4 \mathrm{k}$ with $\sigma$ of 1.46 chains nm ${ }^{-2}$; (c) $3.3 \mathrm{~nm} @ 6 \mathrm{k}$ with $\sigma$ of 1.24 chains nm ${ }^{-2}, 5.6 \mathrm{~nm} @ 6 \mathrm{k}$ with $\sigma$ of 1.31 chains $\mathrm{nm}^{-2}$, and 7.2nm@6k with $\sigma$ of 1.30 chains nm ${ }^{-2}$; (d) 4.6nm@6k with $\sigma$ of 2.25 chains nm ${ }^{-2}$ and $5.6 \mathrm{~nm} @ 6 \mathrm{k}$ with $\sigma$ of 0.87 chains $\mathrm{nm}^{-2}$. The weight fraction of PS ligands of Au@PS NPs was determined by the weight loss during thermal decomposition of the PS ligands until reaching the temperature corresponding to the minimum value of weight (\%). The weight fraction of $\mathrm{Au}$ corresponds to the minimum value of weight $(\%){ }^{1}$ 

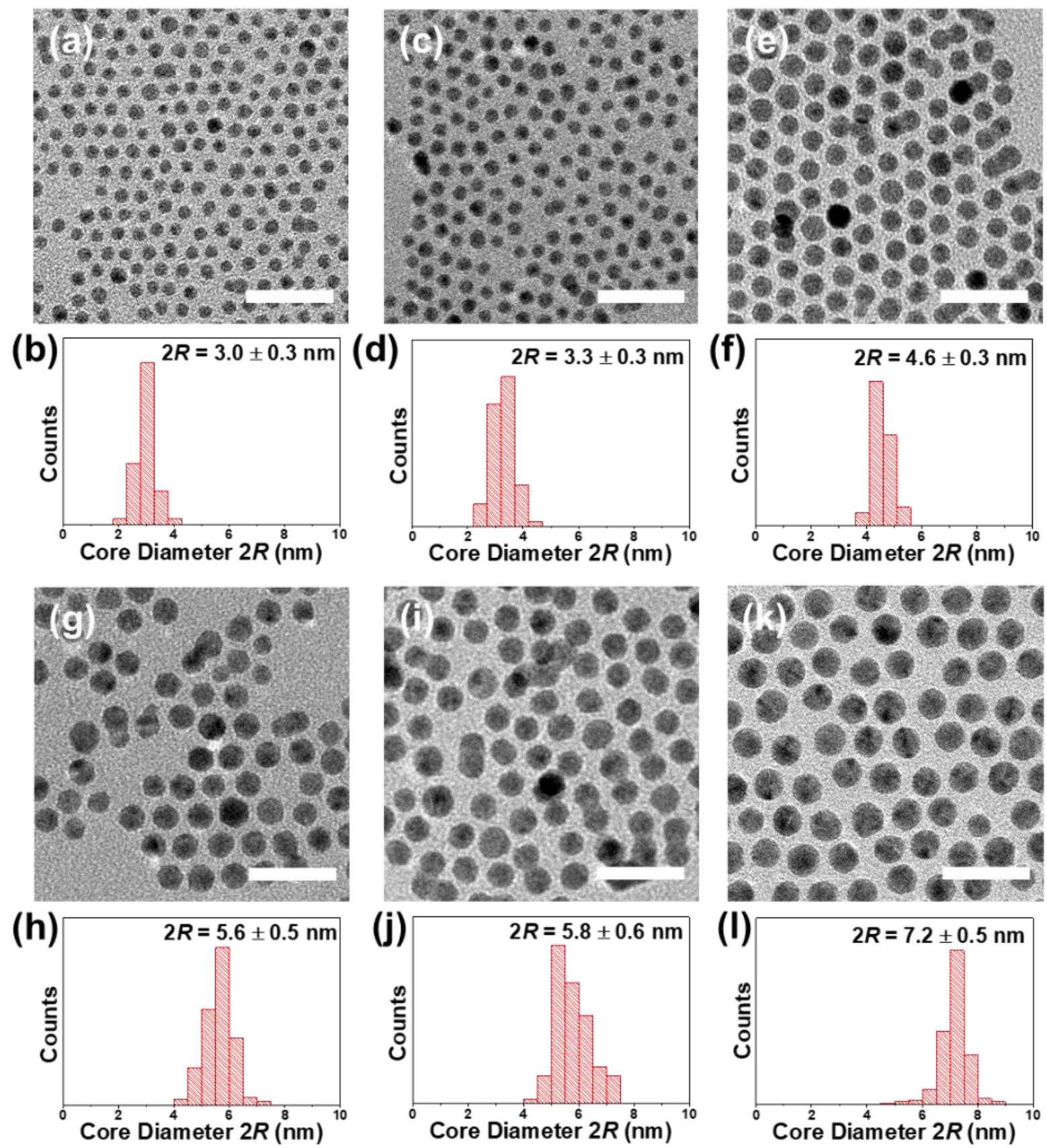

Figure S2. Characterization of as-synthesized Au NPs. TEM images and histograms of Au NPs with diameters $(2 R)$ of $(\mathrm{a}, \mathrm{b}) 3.0 \pm 0.3 \mathrm{~nm}$; (c, d) $3.3 \pm 0.3 \mathrm{~nm}$; (e, f) $4.6 \pm 0.3 \mathrm{~nm}$; (g, h) $5.6 \pm 0.5 \mathrm{~nm}$; (i, j) $5.8 \pm 0.6 \mathrm{~nm}$; and $(\mathrm{k}, 1) 7.2 \pm 0.5 \mathrm{~nm}$. The scale bars are $20 \mathrm{~nm}$. 

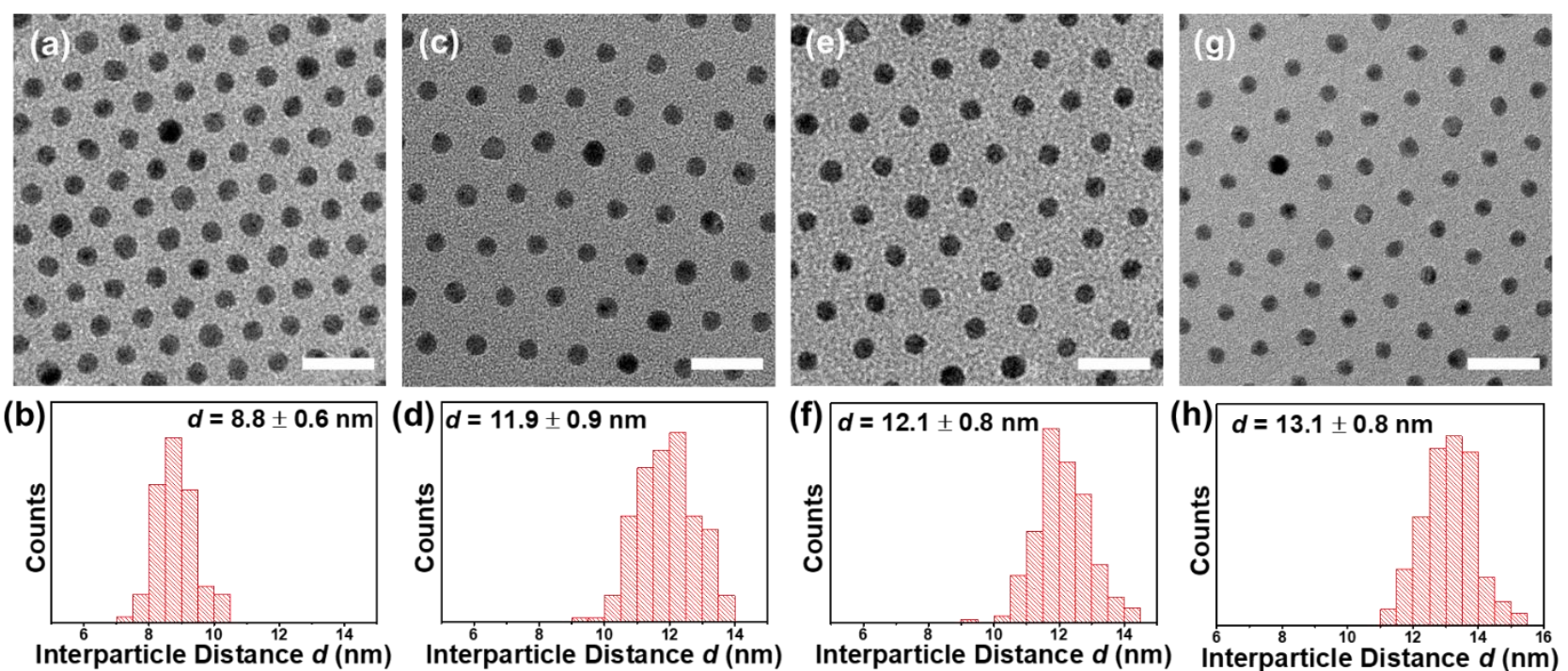

Figure S3. TEM images and histograms of $4.6 \mathrm{~nm} \mathrm{Au} \mathrm{NPs} \mathrm{grafted} \mathrm{by} \mathrm{PS-SH} \mathrm{ligands} \mathrm{with} \mathrm{different}$ molecular weights $\left(M_{n}\right)$ of $(\mathrm{a}, \mathrm{b}) 2 \mathrm{k}$, (c, d) $4 \mathrm{k},(\mathrm{e}, \mathrm{f}) 6 \mathrm{k}$, and $(\mathrm{g}, \mathrm{h}) 8 \mathrm{k}$. The scale bars are $20 \mathrm{~nm}$.

Table S1. Characteristics of various combinations of different PS- $b$-P4VP and 4.6 nm Au NPs grafted by various $M_{\mathrm{n}}$ PS ligands.

\begin{tabular}{|c|c|c|c|c|c|c|c|}
\hline Au@PS & $\begin{array}{c}M_{n} \text { of } \\
\text { ligands } N \\
\left(\mathrm{~kg} \mathrm{~mol}^{-1}\right)\end{array}$ & $\begin{array}{c}\text { Grafting } \\
\text { Density } \sigma \\
\left(\text { chains } \mathbf{n m}^{-2} \text { ) }\right.\end{array}$ & $\begin{array}{c}N_{\text {SDPB }} \\
\left(\mathrm{kg} \mathrm{mol}^{-1}\right)\end{array}$ & $\begin{array}{c}M_{\mathrm{n}} \text { of PS } \\
\text { Blocks } P \\
\left(\mathrm{~kg} \mathrm{~mol}^{-1}\right) \\
\end{array}$ & $P / N$ & $P / N_{\text {SDPB }}$ & Nanostructure \\
\hline \multirow{2}{*}{ 4.6nm@2k } & \multirow{2}{*}{1.8} & \multirow{2}{*}{1.29} & \multirow{2}{*}{0.08} & 3.3 & 1.83 & 41.25 & Crusted Onion \\
\hline & & & & 9.8 & 5.44 & 122.50 & Crusted Onion \\
\hline \multirow{2}{*}{ 4.6nm@4k } & \multirow{2}{*}{4.3} & \multirow{2}{*}{1.37} & \multirow{2}{*}{2.45} & 9.8 & 2.28 & 4.00 & Seeded Onion \\
\hline & & & & 19.0 & 4.42 & 7.76 & Crusted Onion \\
\hline \multirow{2}{*}{ 4.6nm@6k } & \multirow{2}{*}{6.4} & \multirow{2}{*}{1.49} & \multirow{2}{*}{4.37} & 9.8 & 1.53 & 2.24 & ALO \\
\hline & & & & 19.0 & 2.97 & 4.35 & Seeded Onion \\
\hline \multirow{2}{*}{ 4.6nm@8k } & \multirow{2}{*}{8.0} & \multirow{2}{*}{1.58} & \multirow{2}{*}{5.82} & 9.8 & 1.23 & 1.68 & ALO \\
\hline & & & & 19.0 & 2.38 & 3.26 & Seeded Onion \\
\hline
\end{tabular}




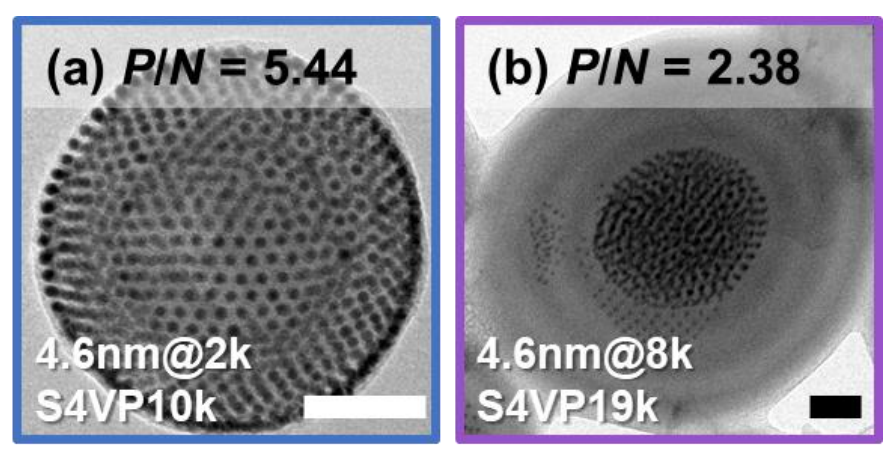

Figure S4. TEM images of PS- $b$-P4VP/Au@PS hybrid particles at a fixed core size of Au NPs (4.6 nm): (a) S4VP10k/4.6nm@2k, (b) S4VP19k/4.6nm@8k. The scale bars are $50 \mathrm{~nm}$.
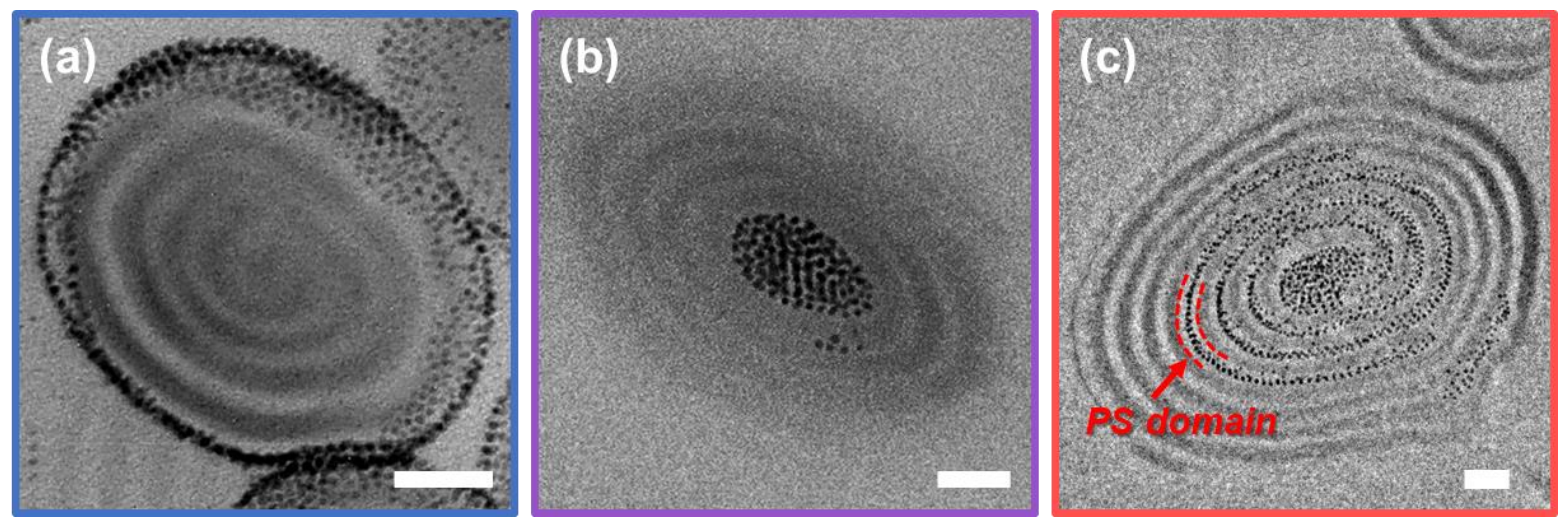

Figure S5. Cross-sectional TEM images of PS- $b-\mathrm{P} 4 \mathrm{VP} / \mathrm{Au} @ \mathrm{PS}$ hybrid particles: (a) S4VP10k/4.6nm@2k, (b) S4VP10k/4.6nm@4k, and (c) S4VP10k/4.6nm@6k. The scale bars are 50 nm. 

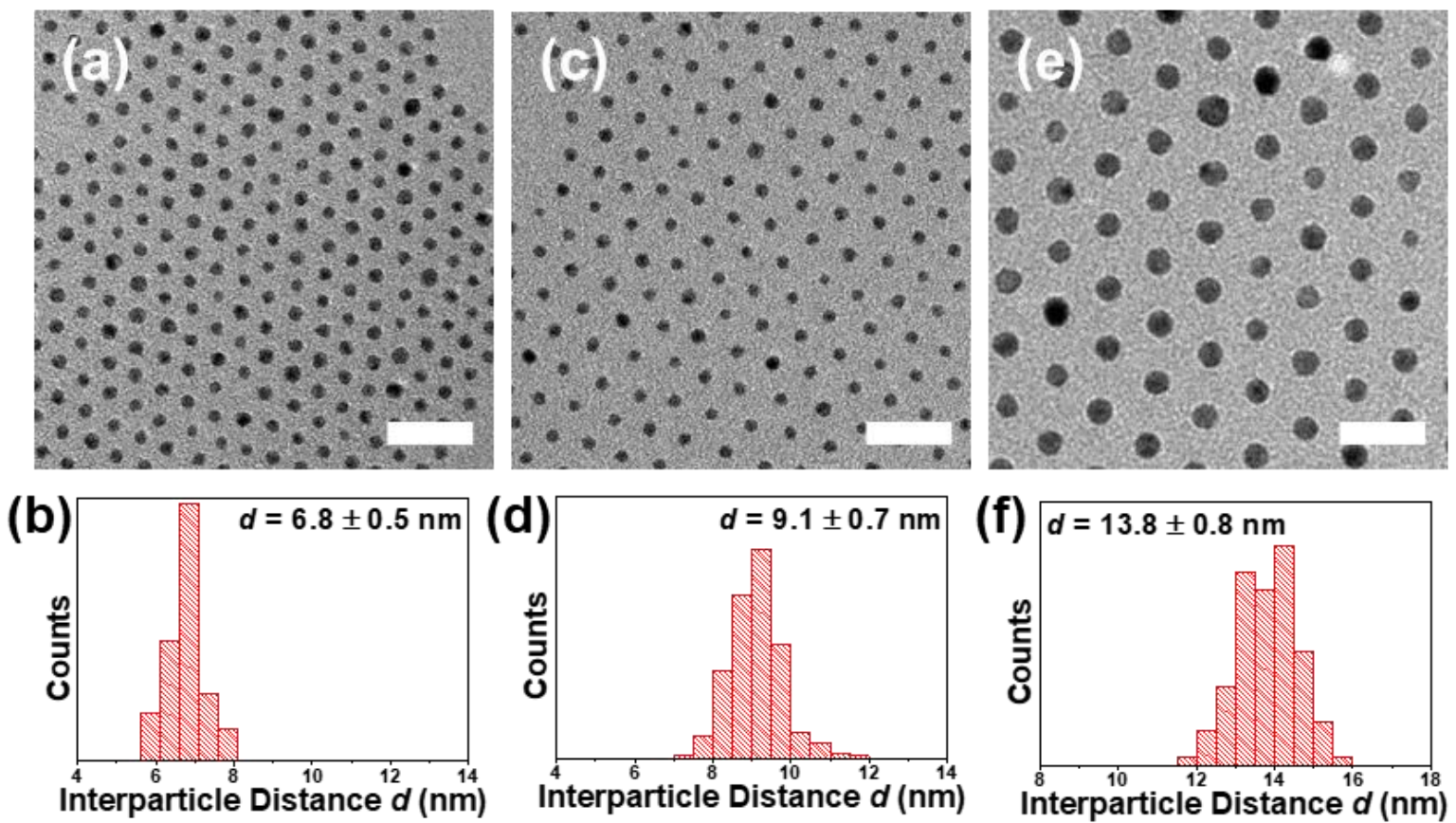

Figure S6. TEM images and histograms of Au@PS NPs: (a, b) 3.0nm@2k, (c, d) 3.0nm@4k, and (e, f) $5.8 \mathrm{~nm} @ 4 \mathrm{k}$. The scale bars are $20 \mathrm{~nm}$.

Table S2. Characteristics of various combinations of PS- $b$-P4VP and Au@PS.

\begin{tabular}{|c|c|c|c|c|c|c|c|}
\hline Au@PS & $\begin{array}{c}M_{\mathrm{n}} \text { of } \\
\text { ligands } N \\
\left(\mathrm{~kg} \mathrm{~mol}^{-1)}\right.\end{array}$ & $\begin{array}{c}\text { Grafting } \\
\text { Density } \sigma \\
\left(\text { chains } \mathbf{n m}^{-2} \text { ) }\right.\end{array}$ & $\begin{array}{c}N_{\mathrm{SDPB}} \\
\left(\mathrm{kg} \mathrm{mol}^{-1}\right)\end{array}$ & $\begin{array}{c}M_{\mathrm{n}} \text { of PS } \\
\text { Blocks } P \\
\left(\mathrm{~kg} \mathrm{~mol}^{-1}\right)\end{array}$ & $P / N$ & $P / N_{\text {SDPB }}$ & Nanostructure \\
\hline 3.0nm@2k & 1.8 & 1.78 & 0.35 & 3.3 & 1.83 & 9.43 & Crusted Onion \\
\hline 3.0nm@4k & 4.3 & 1.56 & 3.05 & 9.8 & 2.28 & 3.21 & ALO \\
\hline 5.8nm@4k & 4.3 & 1.46 & 1.66 & 9.8 & 2.28 & 5.90 & Seeded Onion \\
\hline
\end{tabular}



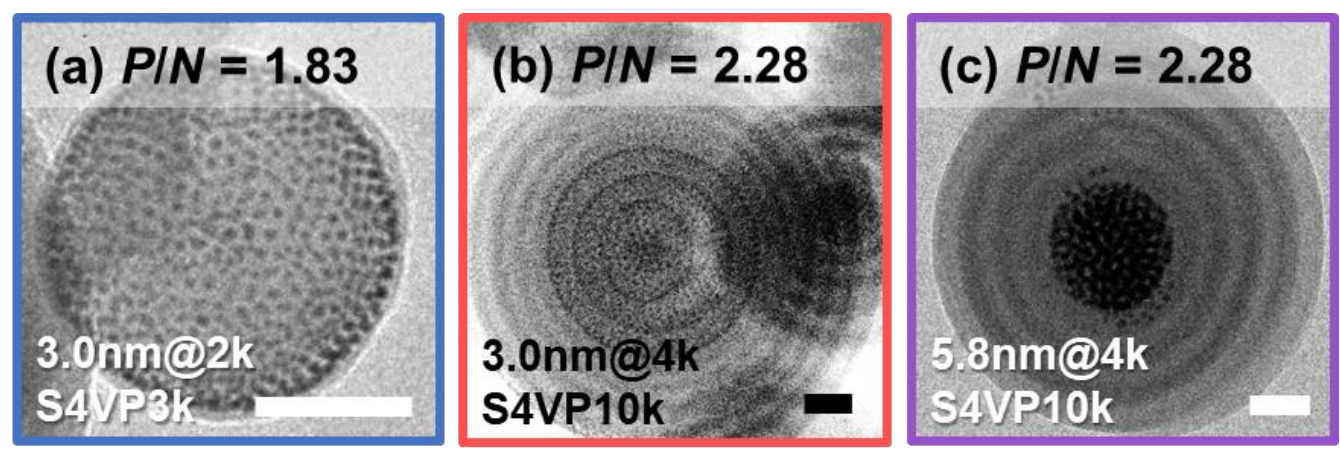

Figure S7. TEM images of PS- $b$-P4VP/Au@PS hybrid particles: (a) S4VP3k/3.0nm@2k, (b) S4VP10k/3.0nm@4k, and (c) S4VP10k/5.8nm@4k. The scale bars are 50 nm.
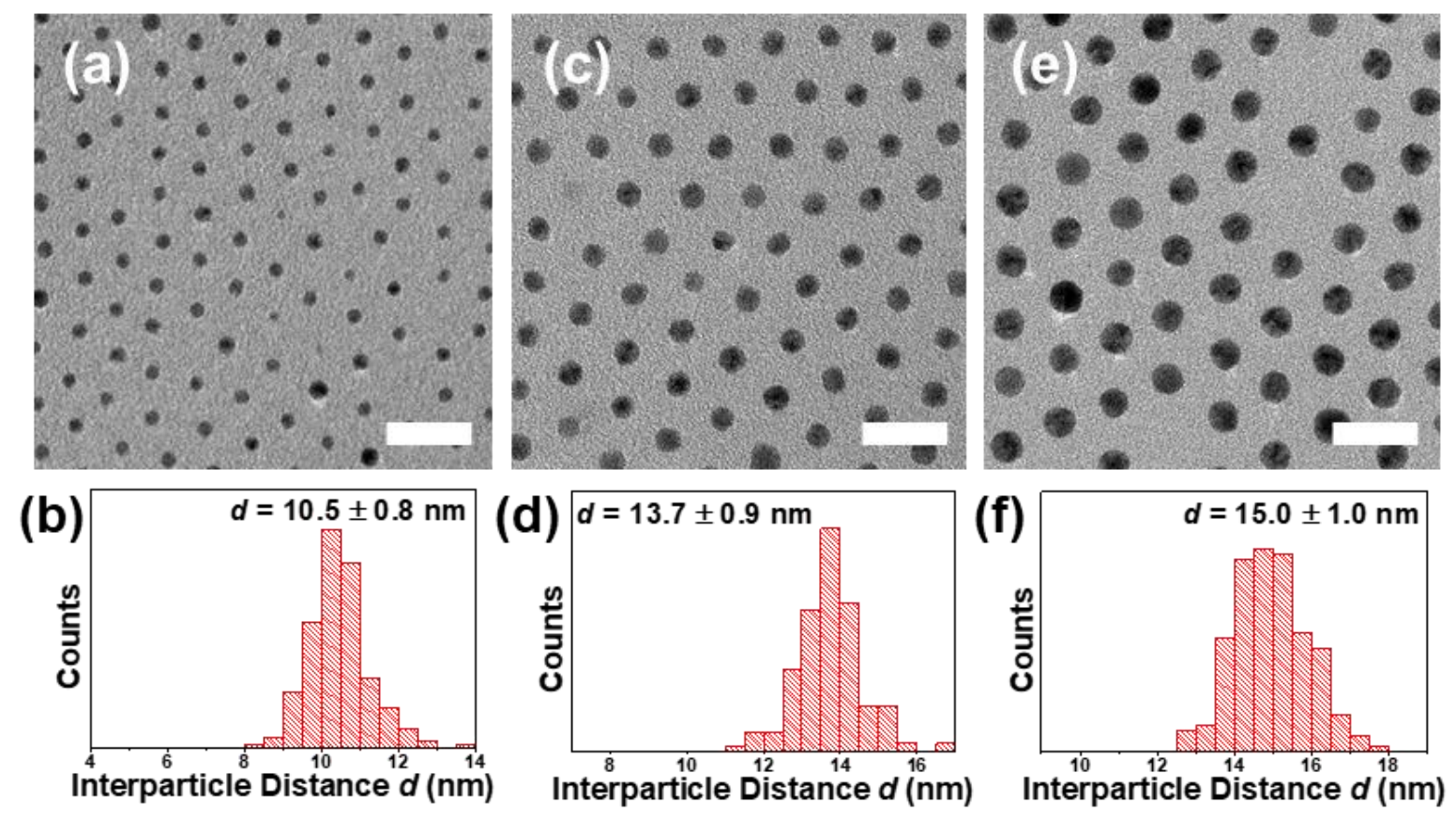

Figure S8. TEM images and histograms of Au@PS NPs: (a,b) 3.3nm@6k, (c,d) 5.6nm@6k, and (e,f) 7.2nm@6k. The scale bars are $20 \mathrm{~nm}$. 
Table S3. Characteristics of various combinations of $\mathrm{PS}_{19 \mathrm{k}}-b-\mathrm{P} 4 \mathrm{VP}_{22 \mathrm{k}}$ and $6 \mathrm{k}$ PS-grafted Au NPs with different core sizes.

\begin{tabular}{|c|c|c|c|c|}
\hline Au@PS & $\begin{array}{l}\text { Grafting Density } \sigma \\
\quad\left(\text { chains } \mathbf{n m}^{-2}\right)\end{array}$ & $N_{\text {SDPB }}\left(\mathrm{kg} \mathrm{mol}^{-1}\right)$ & $P / N_{S D P B}$ & Nanostructure \\
\hline 3.3nm@6k & 1.24 & 5.32 & 3.57 & ALO \\
\hline 5.6nm@6k & 1.31 & 4.17 & 4.56 & Seeded Onion \\
\hline 7.2nm@6k & 1.30 & 3.39 & 5.60 & Crusted Onion \\
\hline
\end{tabular}
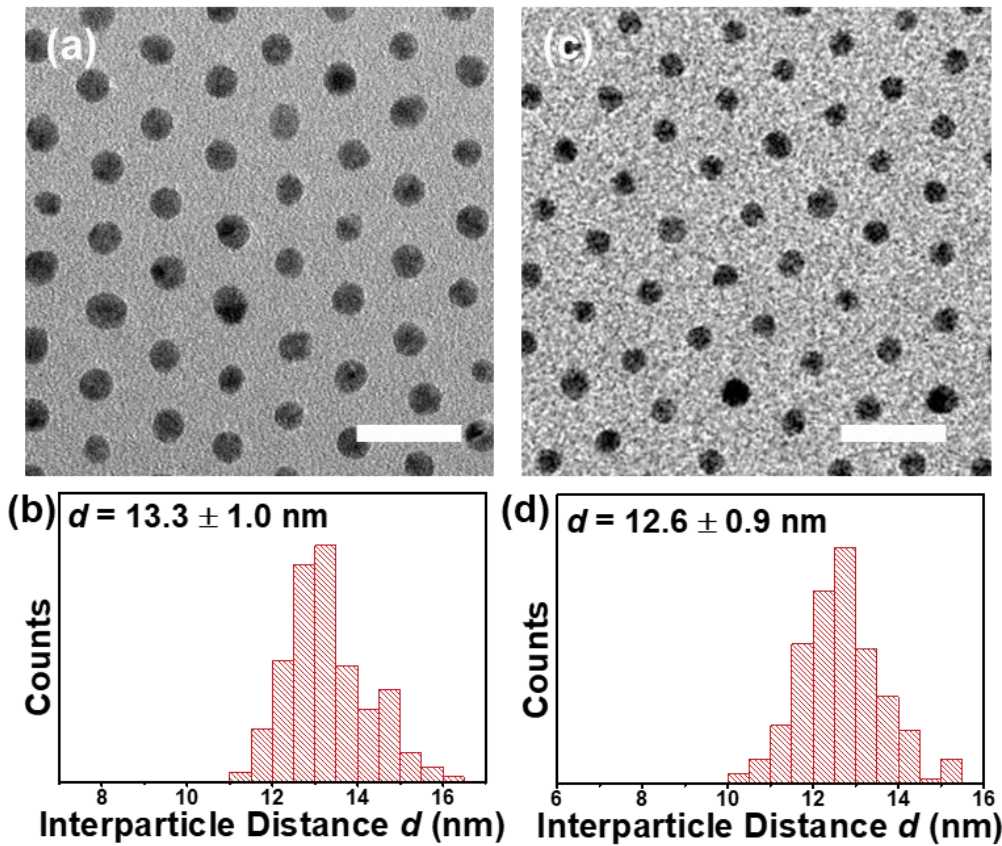

Figure S9. TEM images and histograms of Au@PS NPs with different areal chain density $(\sigma)$ values: (a, b) 5.6nm@6k with $\sigma$ of 0.87 , and (c, d) 4.6nm@6k with $\sigma$ of 2.25 . The scale bars are $20 \mathrm{~nm}$. 
Table S4. Characteristics of combinations of $\mathrm{PS}_{10 \mathrm{k}}-b-\mathrm{P} 4 \mathrm{VP}_{10 \mathrm{k}}$ and grafting density-controlled Au@PS NPs.

\begin{tabular}{|c|c|c|c|c|}
\hline Au@PS & $\begin{array}{l}\text { Grafting Density } \sigma \\
\quad\left(\text { chains } \mathbf{n m}^{-2}\right)\end{array}$ & $N_{\text {SDPB }}\left(\mathrm{kg} \mathrm{mol}^{-1}\right)$ & $P / N_{S D P B}$ & Nanostructure \\
\hline 4.6nm@6k & 1.49 & 4.37 & 2.24 & ALO \\
\hline 4.6nm@6k & 2.25 & 3.24 & 3.02 & Seeded Onion \\
\hline $5.6 \mathrm{~nm} @ 6 \mathrm{k}$ & 0.87 & 5.13 & 1.91 & ALO \\
\hline $5.6 \mathrm{~nm} @ 6 \mathrm{k}$ & 1.31 & 4.17 & 2.35 & Seeded Onion \\
\hline
\end{tabular}
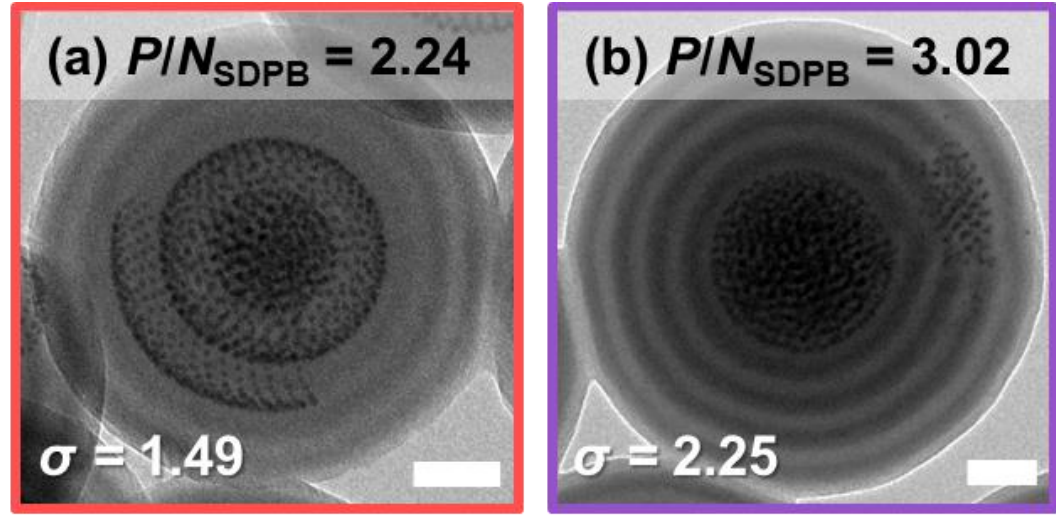

Figure S10. TEM image of the S4VP10k/4.6nm@6k hybrid particles with (a) $\sigma=1.49$, and (b) $\sigma=$ 2.25 chains $\mathrm{nm}^{-2}$. The scale bars are $50 \mathrm{~nm}$. 


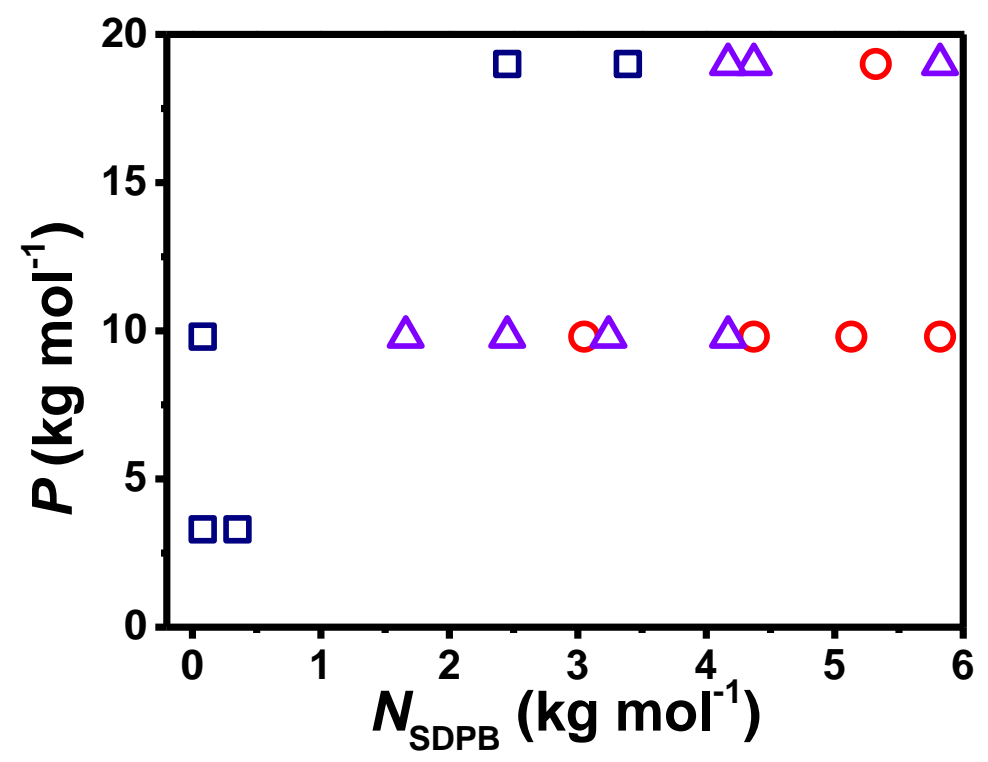

Figure S11. A morphology diagram of the S4VP/Au@PS hybrid particles by plotting $P$ vs. $N_{\text {SDPB }}$. Blue squares $(\square)$, purple triangles $(\triangle)$, and red circles $(\odot)$ represent crusted onion, seeded onion, and ALO structures, respectively.

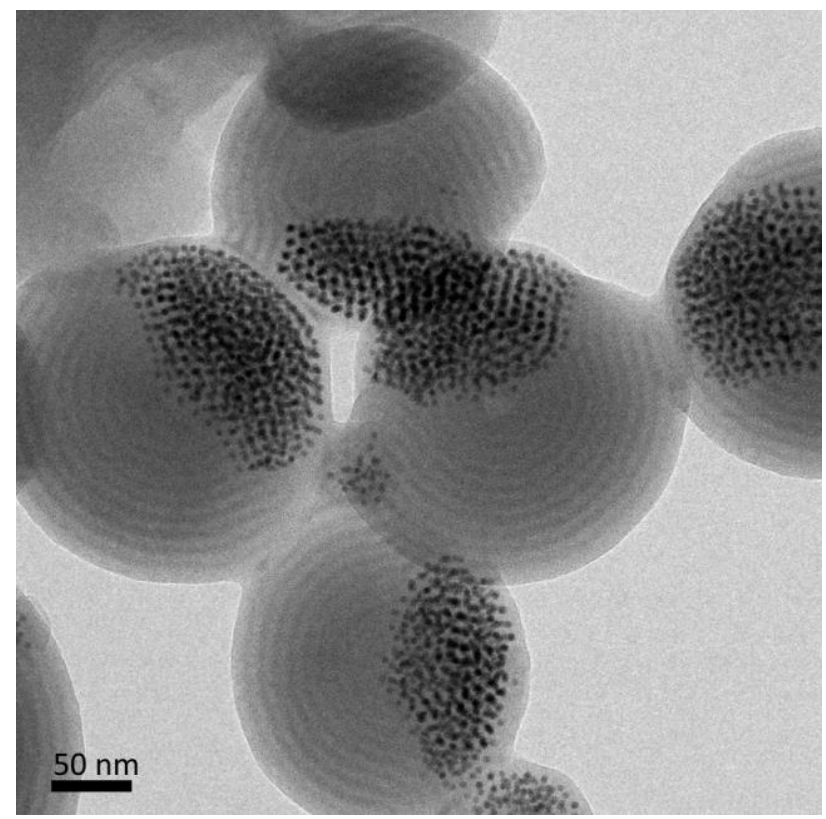

Figure S12. TEM image of S4VP3k/4.6nm@6k hybrid particles. 


\section{References}

(1) Xu, M.; Ku, K. H.; Lee, Y. J.; Shin, J. J.; Kim, E. J.; Jang, S. G.; Yun, H.; Kim, B. J. EntropyDriven Assembly of Nanoparticles within Emulsion-Evaporative Block Copolymer Particles: Crusted, Seeded, and Alternate-Layered Onions. Chem. Mater. 2020, 32, 7036-7043. 